\title{
EXTRAÇÃO POR SOLVENTES SINÉRGICA APLICADA À SEPARAÇÃO NÍQUEL/CÁLCIO COM CYANEX 272 E D2EHPA
}

\author{
M. F. A. SOUZA ${ }^{1}$, A. S. GUIMARÃES ${ }^{2}$ e M. B. MANSUR ${ }^{3}$
}

${ }^{1}$ Universidade Federal de Minas Gerais, Departamento de Engenharia Metalúrgica e de Materiais

${ }^{2}$ Universidade Federal de Minas Gerais, Departamento de Engenharia Metalúrgica e de Materiais

${ }^{3}$ Universidade Federal de Minas Gerais, Departamento de Engenharia Metalúrgica e de Materiais E-mail para contato: marcelsouza107@yahoo.com.br; marcelo.mansur@demet.ufmg.br

RESUMO - A separação entre cálcio e níquel presente em soluções aquosas sulfúricas foi investigada no presente trabalho mediante extração por solventes sinérgica utilizando-se os extratantes organofosforados comerciais D2EHPA e Cyanex 272. Para identificar e quantificar o efeito sinérgico foi utilizada uma solução aquosa contendo cálcio e níquel em condições de concentração idênticas, de modo a minimizar efeitos de competição na fase aquosa. Verificou-se que o uso da mistura Cyanex $272+$ D2EHPA permitiu a separação de cálcio com elevada seletividade frente ao níquel, que permaneceu na solução aquosa, principalmente na faixa de $\mathrm{pH}$ compreendida entre 4,0 e 5,0. Tal melhoria deveu-se ao sinergismo ocorrido entre os dois extratantes estudados. Portanto, a mistura entre extratantes Cyanex 272 e D2EHPA elevou a seletividade e a capacidade extrativa dos metais investigados, sendo o sistema extrator proposto superior que os extratantes usados individualmente.

\section{INTRODUÇÃO}

A purificação industrial de níquel a partir de licores sulfúricos de lixiviação encontra-se atualmente ancorada quase que exclusivamente na utilização do extratante comercial Cyanex 272 (bis(2,4,4-trimetilpentil) ácido fosfínico). Tal fato deve-se basicamente à eficaz separação Ni-Co na presença de impurezas como cobre, zinco e manganês. Apesar disso, um dos problemas encontrados é a deficiência na extração do cálcio, que permanece no licor como contaminante do níquel (Flett, 2005; Guimarães, 2014). Na tentativa de melhorar a separação Ni-Ca, o uso de sistemas extrativos sinérgicos constitui uma alternativa a ser considerada.

Sistemas extrativos sinérgicos são formados misturando-se diferentes extratantes na fase orgânica, com o intuito de melhorar a capacidade extrativa e a seletividade de uma dada separação considerada difícil de ser executada ao se utilizar um único extratante. Trata-se de uma alternativa mais simples e mais econômica do ponto de vista operacional, se comparada ao desenvolvimento de um extratante novo ainda não utilizado comercialmente.

A separação Ni-Co na presença de Ca foi estudada em uma série de trabalhos realizada na 


\section{9 a 22 de outubro de 2014 \\ Florianópolis/SC}

Mintek (África do Sul) focando inicialmente o uso de oximas na separação Ni-Co (Preston, 1982, 1983a,b), posteriormente usando ésteres de piridina-carboxilatos (Preston e du Preez, 1994, 1996), e mais recentemente usando alquil-piridina (Preston e Du Preez, 2000). Apesar dos resultados promissores, a aplicação prática dos sistemas sinérgicos avaliados nesses estudos ficou comprometida pelo fato dos reagentes empregados serem pouco usados comercialmente na separação hidrometalúrgica de metais base.

Em estudos mais recentes, Ndlovu e Mahlangu (2008) comprovaram o sinergismo de uma mistura entre os extratantes comerciais Ácido Versático 10 e LIX84-IC (2-hidroxi-5-nonilacetofenona oxima), em que o $\Delta \mathrm{pH}_{1 / 2}$ da extração Ni-Ca aumentou de 2,10 (Ácido Versático) para 4,75 (mistura). Outras misturas de extratantes também utilizando Ácido Versático 10 mostraram sinergismo na separação Ni-Ca, como é o caso do LIX $^{\circledR} 63$ (5,8-dietil-7-hidroxi-dodecan-6-oxima), utilizando-se o modificador TBP (tributil fosfato) (Cheng, 2006), e do 4PC (4-piridinacaboxilato de n-decila) (Cheng et al., 2010). Em ambos os estudos, observou o efeito sinérgico na separação de níquel frente às impurezas como $\mathrm{Ca}$ e $\mathrm{Mg}$.

Como o Cyanex 272 é o extratante comercial utilizado preferencialmente nas plantas industriais de purificação de níquel, seu uso na composição desses sistemas sinérgicos representaria um diferencial competitivo. O sinergismo da extração Ni-Co foi estudada por Darvishia et al. (2005) utilizando-se o extratante D2EHPA (di(2-etilhexil) ácido fosfórico) ora com Cyanex 272 ora em presença do Cyanex 302 (bis-(2,4,4-trimetilpentil) ácido monotiofosfínico), obtendo-se melhor separação para o sistema D2EHPA/Cyanex 302. Recentemente, Guimarães (2014) observou que a separação Ni-Ca também poderia ser realizada empregando-se misturas entre Cyanex 272 e D2EHPA, logo o objetivo do presente trabalho é avaliar o sinergismo na separação Ni-Ca utilizando-se uma mistura dos extratantes D2EHPA e Cyanex 272.

\section{PROCEDIMENTO EXPERIMENTAL}

\subsection{Reagentes e soluções}

As soluções aquosas sulfúricas foram preparadas diluindo-se os reagentes (grau analítico) $\mathrm{NiSO}_{4} \cdot 6 \mathrm{H}_{2} \mathrm{O}$ (Vetec, 98\%), $\mathrm{CaSO}_{4} \cdot 2 \mathrm{H}_{2} \mathrm{O}$ (Synth, 98\%) e $\mathrm{Li}_{2} \mathrm{SO}_{4} \cdot \mathrm{H}_{2} \mathrm{O}$ (Synth, 99\%) em água deionizada. Para controlar o $\mathrm{pH}$ da fase aquosa foram utilizadas soluções de $\mathrm{NaOH}$ (Vetec, 99\%) e $\mathrm{H}_{2} \mathrm{SO}_{4}$ (Synth, 97\%). A fase orgânica foi preparada diluindo-se os extratantes D2EHPA (di(2etilhexil) ácido fosfórico, Merck, 95\%) e Cyanex 272 (bis(2,4,4-trimetilpentil) ácido fosfínico, Cytec, 85\%) em n-heptano (Synth, 99\%).

\subsection{Ensaios de extração por solventes}

Em um béquer de $500 \mathrm{~mL}$ foram adicionados iguais volumes $(150 \mathrm{~mL})$ de solução aquosa e orgânica. Para minimizar os efeitos de competição na fase aquosa entre os metais cálcio e níquel pelo(s) extratante(s) e realçar o efeito sinérgico da fase orgânica, utilizou-se uma solução aquosa com concentração inicial de $\mathrm{Ca}$ e Ni igual a 0,05 mol. $\mathrm{L}^{-1}$ para cada metal. Ainda, com intuito de manter a força iônica da solução aquosa constante durante os testes, evitando assim a variação no equilíbrio de 
extração dos metais $\mathrm{Ca}$ e $\mathrm{Ni}$, adicionou-se lítio à solução aquosa na concentração de 0,124 mol.L $\mathrm{L}^{-1}$. Com base na literatura, lítio não é extraído por nenhum dos extratantes estudados na faixa de $\mathrm{pH}$ dos ensaios. As fases aquosa e orgânica foram agitadas a $400 \mathrm{rpm}$ utilizando-se um agitador magnético por 5 minutos. Esse tempo é suficiente para que as reações de extração atinjam a condição de equilíbrio químico, com base em estudos anteriores (Mansur et al., 2002; Morais e Mansur, 2004; Mansur e Dorella, 2007; Mantuano et al., 2008; Mansur et al., 2008; Guimarães, 2014). Durante a agitação, fez-se o controle de $\mathrm{pH}$ mediante adição de gotas de solução aquosa de $\mathrm{NaOH}$ ou $\mathrm{H}_{2} \mathrm{SO}_{4}$. Após cessar a agitação, o sistema foi deixado em repouso por 2 minutos para obter a separação entre as fases. Alíquotas de $10 \mathrm{~mL}$ da fase aquosa foram retiradas para análise química em diferentes valores de $\mathrm{pH}$, variando entre 3 e 7 , com incrementos de aproximadamente 0,5. Os ensaios foram realizados a temperatura constante $\left(25 \pm 1^{\circ} \mathrm{C}\right)$. Além do $\mathrm{pH}$, avaliou-se a extração variando-se a composição da fase orgânica, contendo $0,05 \mathrm{~mol} \cdot \mathrm{L}^{-1}$ dos extratantes nas seguintes formulações: (i) D2EHPA, (ii) Cyanex 272, e (iii) D2EHPA + Cyanex 272.

\subsection{Análise química}

As amostras da fase aquosa coletadas nos experimentos de extração por solventes foram analisadas por Espectroscopia de Absorção Atômica, a fim de determinar a concentração de cada metal (cálcio, lítio e níquel) na solução aquosa. A percentagem de extração (\%E) de cada metal para a fase orgânica foi calculada através do balanço de massa entre a fase aquosa inicial e a fase aquosa após a extração, naquele valor de $\mathrm{pH}$. A concentração de metal na fase orgânica foi obtida por balanço de massa.

\section{RESULTADOS E DISCUSSÃO}

Curvas de extração dos metais empregando-se uma solução aquosa sulfúrica contendo Ni $(0,05$ mol.L $\left.\mathrm{L}^{-1}\right)$, Ca $\left(0,05\right.$ mol.L $\left.\mathrm{L}^{-1}\right)$ e Li $\left(0,124\right.$ mol.L $\left.\mathrm{L}^{-1}\right)$ após contactação com fases orgânicas contendo D2EHPA (0,05 mol. $\left.\mathrm{L}^{-1}\right)$, Cyanex $272\left(0,05 \mathrm{~mol} . \mathrm{L}^{-1}\right)$ e a mistura D2EHPA + Cyanex 272 (cada extratante com 0,05 mol. $\mathrm{L}^{-1}$ ) encontram-se mostradas na Figura 1. Em nenhum dos ensaios foi observada a extração significativa de lítio $\left(\% \mathrm{E}_{\mathrm{Li}}<5 \%\right)$ pelos extratantes organofosforados utilizados no estudo, corroborando os resultados de Bukowsky et al. (1992) e Mantuano et al. (2008). Também não foi observada a formação de terceira fase nas condições operacionais estudadas, o que justifica a não utilização de agentes modificadores na formulação das fases orgânicas. 


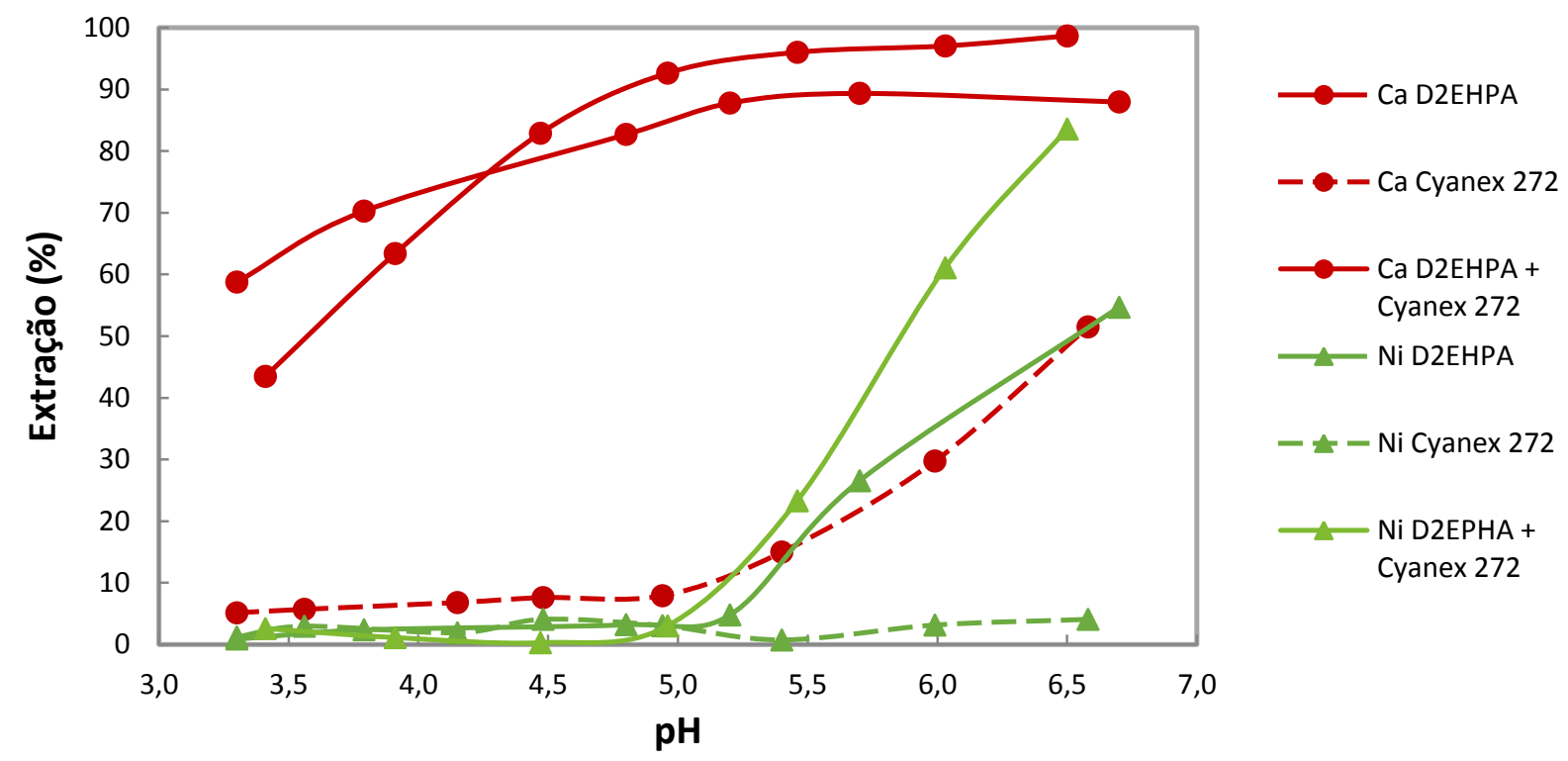

Figura 1 - Extração de níquel e cálcio $\left(0,01\right.$ mol. $\mathrm{L}^{-1}$ cada) por D2EHPA $\left(0,05 \mathrm{~mol} . \mathrm{L}^{-1}\right)$, Cyanex 272 $\left(0,05\right.$ mol. $\left.\mathrm{L}^{-1}\right)$ e mistura: D2EHPA+Cyanex $272\left(0,05\right.$ mol. $\mathrm{L}^{-1}$ cada) diluídos em n-heptano, a $25^{\circ} \mathrm{C}$.

Observa-se na Figura 1 que não há extração de níquel pelo Cyanex $272 \mathrm{em}$ praticamente toda a faixa de $\mathrm{pH}$ estudada, permanecendo o metal em solução aquosa. Essa característica é vantajosa do ponto de vista econômico na purificação comercial de níquel por demandar menor consumo de extratante, já que o metal encontra-se em concentrações elevadas nos licores industriais, normalmente acima de 80 g.L $\mathrm{L}^{-1}$. Com relação à extração de cálcio com Cyanex 272, observa-se que é praticamente inexistente até $\mathrm{pH} 5,0$, valor a partir do qual se verifica o início de sua extração, atingindo cerca de $50 \%$ em pH 6,5. Essa condição operacional, porém, é proibitiva devido à formação de terceira fase em licores industriais quando o pH supera valores iguais a 6,0 (Guimarães, 2014). Portanto, esse limite operacional deve ser obedecido, o que torna a separação Ca-Ni com Cyanex 272 uma operação delicada.

Utilizando-se o extratante D2EHPA, porém, observa-se um aumento considerável na extração de cálcio, mesmo em valores de $\mathrm{pH}$ mais baixos, atingindo cerca de $90 \%$ em pH próximo a 5,2, enquanto o níquel é praticamente mantido em solução aquosa $\left(\% \mathrm{E}_{\mathrm{Ni}}<5 \%\right)$. A partir desse valor de $\mathrm{pH}$, a extração de níquel aumenta consideravelmente, atingindo cerca de 50\% em pH próximo a 6,5. O deslocamento para a esquerda nas curvas de cálcio e níquel com D2EHPA deve-se ao caráter mais fortemente ácido da molécula do extratante, que é derivado do ácido fosfórico, enquanto que o Cyanex 272 é derivado do ácido fosfínico.

Com a mistura dos extratantes D2EHPA e Cyanex 272, praticamente não há extração de níquel em $\mathrm{pH} \leq 5,0$, sendo essa favorecida consideravelmente a partir desse valor. Já a extração de cálcio apresenta comportamento semelhante ao sistema contendo somente D2EHPA, com extração desfavorecida em $\mathrm{pH}<4,5$, porém favorecida a partir desse limite. $\mathrm{O} \mathrm{pH}_{1 / 2}$ relativo à extração de cálcio é próximo a 3,7 e em valores de pH acima de 5 tem-se extrações superiores a $95 \%$ do cálcio. 
Com isso, observa-se uma janela de extração seletiva compreendida entre 4,5 $\leq \mathrm{pH} \leq 5,0$, que favorece a separação $\mathrm{Ca}-\mathrm{Ni}$.

A extração preferencial observada do cálcio frente ao níquel utilizando-se os extratantes organofosforados Cyanex 272 e D2EHPA corrobora os resultados encontrados por Flett (2005) e Guimarães (2014). Tal preferência pela extração de cálcio frente ao níquel tanto pelos extratantes isoladamente quanto pela mistura entre eles, pode ser mensurada ao se analisar os fatores de separação $\mathrm{Ca} / \mathrm{Ni}$ mostrados na Tabela 1 . Observa-se que o extratante D2EHPA é mais seletivo ao cálcio que o Cyanex 272 em praticamente toda a faixa de $\mathrm{pH}$ estudada. Já a mistura D2EHPA + Cyanex 272 mostrou-se muito mais seletivo ao cálcio frente ao Cyanex 272, porém restrito à faixa de $\mathrm{pH}$ compreendida entre 4 e 5, sendo altamente seletivo em $\mathrm{pH}=4,5$.

Tabela 1 - Fator de Separação entre cálcio e níquel em função do pH da fase aquosa $\left(\beta_{\mathrm{Ca} / \mathrm{Ni}}\right)$

\begin{tabular}{c|c|c|c}
\hline \multirow{2}{*}{$\mathbf{p H}$} & \multicolumn{3}{|c}{ Fator de Separação $\left(\boldsymbol{\beta}_{\mathrm{Ca} / \mathrm{Ni}}\right)$} \\
\cline { 2 - 4 } & D2EHPA & Cyanex 272 & $\mathbf{D 2 E H P A}+$ Cyanex 272 \\
\hline 3,3 & 158,9 & 4,4 & 29,1 \\
\hline 3,5 & 96,4 & 2,0 & 31,2 \\
\hline 4,0 & 116,1 & 3,8 & 153,7 \\
\hline 4,5 & 92,0 & 1,9 & 1742,0 \\
\hline 5,0 & 121,0 & 2,7 & 406,0 \\
\hline 5,5 & 198,6 & 24,1 & 79,5 \\
\hline 6,0 & 15,8 & 13,0 & 21,0 \\
\hline 6,5 & 7,7 & 25,0 & 14,6 \\
\hline
\end{tabular}

A Tabela 2 apresenta os valores do fator de sinergismo $\left(\mathrm{FS}_{\mathrm{M}}\right)$ calculado para cada nível de $\mathrm{pH}$. $\mathrm{O}$ fator de sinergismo é a relação entre o coeficiente de distribuição do metal $\mathrm{M}$ no sistema de extração sinérgica [ $\left.\mathrm{D}_{\mathrm{M}}(\mathrm{D} 2 \mathrm{EHPA}+\mathrm{Cyanex} 272)\right]$ e a soma dos coeficientes de distribuição do mesmo metal $M$ nos sistemas com cada extratante individualmente $\left[\mathrm{D}_{\mathrm{M}}(\mathrm{D} 2 \mathrm{EHPA})+\mathrm{D}_{\mathrm{M}}(\mathrm{Cyanex} 272)\right]$ sendo dado por:

$$
F S_{M}=\frac{\left[D_{M}(D 2 E H P A+C y a n e x 272)\right]}{\left[D_{M}(D 2 E H P A)+D_{M}(\text { Cyanex } 272)\right]}
$$

O fator de sinergismo conforme calculado pela equação (1) é usado para determinar a ocorrência do efeito sinérgico de uma mistura de extratantes para um metal $\mathrm{M}$ em particular. A razão entre dois fatores de sinergismo calculados para metais distintos serve como inferência de ocorrência de sinergismo entre esses metais. Da mesma forma que no fator de separação, quanto maior o valor de $\mathrm{FS}_{\mathrm{M}}$ maior é o efeito sinérgico provocado pela mistura de extratantes no metal M (ou na razão entre dois metais). Caso $\mathrm{FS}_{\mathrm{M}}$ seja inferior à unidade, tem-se um efeito sinérgico inverso ou antagonismo. Assim, observa-se, com base nos valores apresentados na Tabela 2, que o efeito sinérgico da mistura 
Cyanex 272 e D2EHPA ocorreu para cálcio em $\mathrm{pH} \geq 4,5$, enquanto para o níquel este ocorreu somente em $\mathrm{pH} \geq 5,5$. No que tange à separação $\mathrm{Ni}-\mathrm{Ca}$, porém, tem-se forte efeito sinérgico na faixa compreendida entre 4,0 $\leq \mathrm{pH} \leq 5,0$, confirmando ser essa faixa de $\mathrm{pH}$ a mais adequada para a pretendida separação entre cálcio e níquel.

Tabela 2 - Fator de Sinergismo para cálcio e níquel em função do pH

\begin{tabular}{c|c|c|c}
\hline \multirow{2}{*}{$\mathbf{p H}$} & \multicolumn{3}{|c}{ Fator de Sinergismo } \\
\cline { 2 - 4 } & $\mathbf{N i}$ & $\mathbf{C a}$ & $\mathbf{C a} / \mathbf{N i}$ \\
\hline 3,3 & 1,2 & 0,5 & 0,4 \\
\hline 3,5 & 0,5 & 0,6 & 1,2 \\
\hline 4,0 & 0,3 & 0,6 & 2,3 \\
\hline 4,5 & 0,0 & 1,7 & 43,7 \\
\hline 5,0 & 0,5 & 3,1 & 6,4 \\
\hline 5,5 & 7,9 & 3,8 & 0,5 \\
\hline 6,0 & 2,7 & 3,7 & 1,3 \\
\hline 6,5 & 4,9 & 8,5 & 8,5 \\
\hline
\end{tabular}

\section{CONCLUSÕES}

A separação Ni-Ca através da extração por solventes sinérgica utilizando a mistura dos extratantes comerciais Cyanex 272 e D2EHPA foi estudada neste trabalho em condições operacionais tais que permitissem a minimização dos efeitos de competição entre os metais na fase aquosa. Os resultados demonstraram, com base nos fatores de separação $\mathrm{Ni}-\mathrm{Ca}$, que houve significativa melhora na separação de cálcio frente ao níquel ao se empregar a mistura entre esses reagentes, na faixa de $\mathrm{pH}$ compreendida entre 4 e 5 . A explicação para essa melhoria deve-se ao sinergismo ocorrido na fase orgânica. Ambos extratantes usados são reagentes derivados de organofosforados largamente usados comercialmente na extração de metais base, o que confere ao sistema proposto uma maior probabilidade de uso em escala industrial. Estudos aprofundados de caracterização analítica das espécies envolvidas são sugeridos para melhor compreender a química reacional ocorrida e inferir sobre a interação entre os extratantes usados na fase orgânica.

\section{AGRADECIMENTOS}

Os autores agradecem ao CNPq, CAPES, FAPEMIG e INCT-Acqua pelo apoio à pesquisa.

\section{REFERÊNCIAS}

BUKOWSKY, H.; GLOEB, K.; UHLEMANN, E.; MÜHL, P. The separation of calcium and magnesium from lithium chloride by liquid-liquid extraction with di(2-ethylhexyl) phosphoric acid. Hydrometallurgy, v. 28, 323-329, 1992. 


\section{9 a 22 de outubro de 2014 \\ Florianópolis/SC}

CHENG, C.Y. Solvent extraction of nickel and cobalt with synergistic systems consisting of carboxylic acid and aliphatic hydroxyoxime. Hydrometallurgy, v. 84, 109-117, 2006.

CHENG, C.Y.; BODDY, G.; ZHANG, W.; GODFREY, M.; ROBINSON, D.J.; PRANOLO, Y.; ZHU, Z.; WANG, W. Recovery of nickel and cobalt from laterite leach solutions using direct solvent extraction: Part 1 - selection of a synergistic SX system. Hydrometallurgy, v. 104, 45$52,2010$.

DARVISHIA, D.; HAGHSHENASA, D.F.; ALAMDARIB, E. K.; SADRNEZHAADA, S. K.; HALALIA, M. Synergistic effect of Cyanex 272 and Cyanex 302 on separation of cobalt and nickel by D2EHPA. Hydrometallurgy, v. 77, p. 227-238, 2005.

FLETT, D.S. Solvent extraction in hydrometallurgy: the role of organophosphorus extractants. Journal of Organometallic Chemistry, v. 609, p. 2426-2438, 2005.

GUIMARÃES, A.S. Purificação de níquel a partir de soluções sulfúricas por extração por solventes utilizando-se Cyanex 272 e mistura de extratantes. Dissertação de Mestrado. Universidade Federal de Minas Gerais, BH, MG, Brasil, 144p., 2014.

MANSUR, M.B.; DORELLA, G. A Study of the Separation of Cobalt from Spent Li-ion Batteries Residues. Journal of Power Sources, v. 170, p. 210-215, 2007.

MANSUR, M.B.; MAGALHÃES, F.S.; ROCHA, S.D.F.; BENEDETTO, J.S. Selective Extraction of Zinc(II) over Iron(II) from spent hydrochloric acid pickling effluents by liquid-liquid extraction. Journal of Hazardous Materials, v. 150, p. 669-678, 2008.

MANSUR, M.B.; SLATER, M.J.; BISCAIA Jr., E.C. Equilibrium analysis of the reactive liquidliquid test system ZnSO4/D2EHPA/n-heptane. Hydrometallurgy, v. 63, p. 107-116, 2002.

MANTUANO, D.P.; DORELLA, G.; ELIAS, R.C.A.; MANSUR, M.B. Analysis of a hydrometallurgical route to recover base metals from spent rechargeable batteries by liquidliquid extraction with Cyanex 272. Journal of Power Sources, v. 159, p. 1510-1518, 2008.

MORAIS, B.S.; MANSUR, M.B. Characterization of the reactive test system ZnSO4/D2EHPA in nheptane. Hydrometallurgy, v. 74, p. 11-18, 2004.

NDLOVU, B.; MAHLANGU, T. Calcium and magnesium rejection from sulphate solutions in lateritic nickel solvent extraction using Versatic 10 acid-Lix ${ }^{\circledR} 84$ IC system. Template Journal, v. 108, p. 223-227, 2008.

PRESTON, J. Solvent extraction of base metals by mixtures of organophosphoric acids and nonchelating oximes. Hydrometallurgy, v. 10, p. 187-204, 1983 a.

PRESTON, J. Solvent extraction of nickel and cobalt by mixtures of organophosphoric acids and nonchelating oximes. Hydrometallurgy, v. 11, p. 105-124, $1983 \mathrm{~b}$. 
PRESTON, J. Solvent extraction of nickel and cobalt by organophosphorous acids. I. Comparison of phosphoric, phosphonic and phosphinic acid systems. Hydrometallurgy, v. 9, p. 115-183, 1982.

PRESTON, J.; DU PREEZ, A. Separation of nickel and calcium by solvent extraction using mixtures of carboxylic acids and alkylpiridines. Hydrometallurgy, v. 58, p. 239-250, 2000.

PRESTON, J.; DU PREEZ, A. Solvent extraction of nickel from acidic solutions using synergistic mixtures containing pyridinecarboxylate esters. Part 1. Systems based on organophosphorous acids. J. Chem. Tech. Biotechol., v. 66(1), p. 86-94, 1996.

PRESTON, J.; DU PREEZ, A. Synergistic effects in the solvent extraction of some divalent metals by mixtures of Versatic acid and pyridine carboxylate esters. J. Chem. Tech. Biotechol., v. 61 (2), p. 159-166, 1994. 